marsupials? . Lactational amenorrhoea may similarly involve mammary reflex control of pituitary hormones, in this case inhibition of follicle-stimulating hormone and luteinizing hormone release. Withdrawal of large quantitites of glucose and other substrates for milk synthesis from the bloodstream leads to changes in levels of hormones that regulate blood concentration of these solutes, such as insulin and glucagon.

However, Peaker and Maule Walker observed no difference between lactating and non-lactating non-mastectomized goats in fertility or in oestrous cycle periodicity. Furthermore, the mastectomized goats exhibited oestrous disorders and impaired fertility before they had ever become pregnant or lactated. These facts suggest that interruption of lactation is not responsible for the reproductive disorders associated with mastectomy. A more likely explanation comes from new evidence that non-lactating mammary gland releases hormones into the systemic circulation, in addition to producing local hormones such as prostaglandins during lactation. From earlier observations by Linzell and colleagues $^{8-10}$ that the mammary gland of goat can metabolize steroid hormones, Maule Walker and Peaker ${ }^{1}$ have gone on to find that the rise in plasma concentration of oestradiol- $17 \beta$ during the last four days before term is due almost entirely to increased production of the hormone by mammary gland.

It remains to be seen whether reproductive disorders in mastectomized goats are related to this mammary production of oestradiol-17 $\beta$, or of other known steroid, peptide, or protein hormones, or of 'new hormones', or else to a non-hormonal mammary signal. The great variability among mammals in the control of reproduction suggests that the goat is not necessarily a universal model and studies on other mammalian species, especially on humans, are now urgently needed.

1. Maule Walker \& Peaker J. Physiol., Lond. 284, 71 (1978).

2. Peaker \& Maule Walker Nature 284, 165 (1980).

3. Maule Walker \& Peaker J. Physiol., Lond. 312, 63 (1980)

4. Peaker in Hormones and Metabolism in Ruminants (ed Forbes \& Lomax) 122 (Agricultural Research Council, London, 1981)

5. Marshall \& Kirkness Biochem J. 2, 1 (1907)

6. Linzell Q. Jl exp. Physiol. 48, 34 (1963).

7. Renfree Nature 278, 549 (1979).

8. Slotkin et al. Nature 225, 385 (1970).

9. Challis \& Linzell $J$. Endocr. 57, 451 (1973).

10. Heap et al. J. Endocr. 64, 485 (1975)

\title{
Exciting Rydberg atoms in cavities
}

\section{from Peter Knight}

HighLY excited atoms with one electron orbiting at large distances from an unexcited core are termed Rydberg atoms and have remarkable properties. Their size gives them a large polarizability, and they are easy to ionize in weak electric fields. If they possess a large angular momentum they do not return rapidly to their ground state by radiative decay simply because decay photons cannot easily carry away all that excess angular momentum. Rydberg atoms provide a unique vehicle for studying radiative processes over long times. In mid 1981, Kleppner (Phys. Rev. Lett. 47, 233; 1981) proposed using such atoms to study the effects of cavity mode confinement in partially 'freezing out' spontaneous decay rates (Nature 293, 514; 1981). A recent experiment by Vaidyanathan, Spencer and Kleppner (Phys. Rev. Lett. 47, 1592; 1981) reports the observation of such waveguide effects, not on spontaneous transitions but on transitions induced in the Rydberg atoms by thermal blackbody radiation.

Rydberg atoms have been used before as detectors of far-IR and microwave blackbody radiation (Nature 279, 476; 1979) but only in effectively unbounded space. Kleppner's new experiment reports a sudden increase in the induced lifetime of a Peter Knight is in the Optics Section, The Blackett Laboratory, Imperial College, Prince Consort Road, London SW7 2BZ.

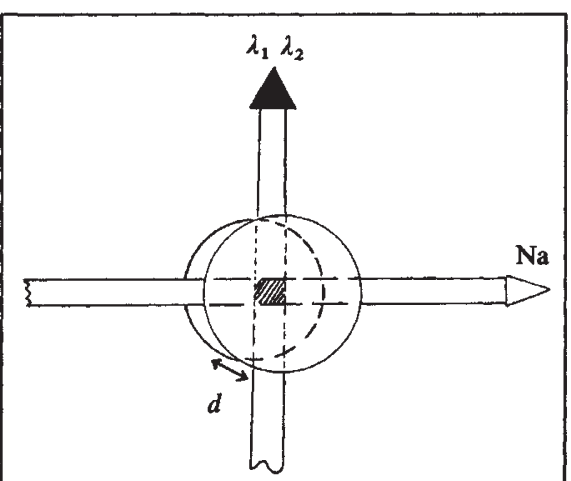

Fig.1 Schematic outline of experiment in which a sodium atomic beam is excited by lasers of wavelength $\lambda_{1}, \lambda_{2}$ to the $29 \mathrm{~d}$ state between two conducting plates.

Rydberg atomic transition between two conducting plates when the resonant wavelength is increased beyond the waveguide cut-off (twice the plate separation $d$ ). The conducting plates are unable to support field modes of wavelength less than $2 d$ in the direction normal to the plates. In addition, those modes parallel to the plates have their density altered but not turned off. Thermal radiation can only be supported on these modes so that transitions induced by blackbody radiation inside the plates are sensitive to the confining geometry.

The technique used to observe these waveguide effects by Kleppner and his

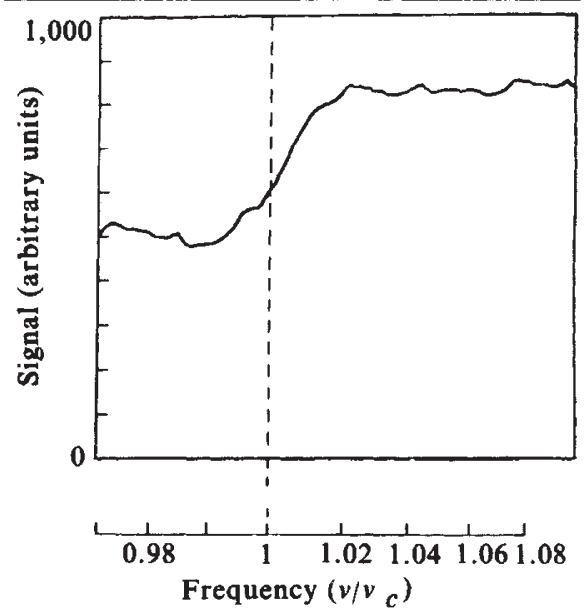

Fig.2 Blackbody radiative transfer signals $29 \mathrm{~d}-30 \mathrm{p}$ in sodium between parallel conducting plates as the transition frequency $v$ is Stark tuned through the cut-off frequency $\nu_{c}=c / 2 d$ (from Vaidyanathan et al.).

group is particularly ingenious and is outlined schematically in Fig. 1. The sodium $29 \mathrm{~d}$ Rydberg state is populated by stepwise absorption from two pulsed lasers of wavelengths $\lambda_{1}$ and $\lambda_{2}$ which drive the transitions $3 \mathrm{~s}$ to $3 \mathrm{p}$ to $29 \mathrm{~d}$ in an atomic beam travelling between two conducting plates separated by $1 / 3 \mathrm{~cm}$. The whole apparatus is placed in a shielded box at $180 \mathrm{~K}$ so that the photon occupation number at wavelength $\lambda \sim 2 d$ is approximately 86 . All polarizations in the transition are possible. The transition $29 \mathrm{~d}$ to $30 \mathrm{p}$ at a wavelength of $2 / 3 \mathrm{~cm}(\sim 2 d)$ is chosen for study and is excited by the thermal field at a rate of 300 per second. Transitions to other levels at $\lambda$ $\neq 2 d$ are of course also induced and contribute to a $d$-insensitive background.

The plate separation is kept fixed but the transition wavelength is varied by exploiting the large polarizability of Rydberg states to Stark-shift energy levels in a small d.c. voltage across the plates. The cut-off frequency $v_{c}=c / 2 d$ is $1.48 \mathrm{~cm}^{-1}$. As the electric field across the plates is varied from 0.7 to $5.7 \mathrm{~V} \mathrm{~cm}^{-1}$, the transition frequency varies from $0.97 v_{c}$ to $1.11 v_{c}$. The transition rate for the production of $30 \mathrm{p}$ atoms is measured by selectively ionizing only those $30 \mathrm{p}$ atoms making the transition, and counting the ions. As the transition frequency $v$ approaches the waveguide cutoff frequency $v_{c}$, the rate of transition suddenly changes (Fig. 2): on the righthand side, blackbody photons can fit between the plates, and on the left-hand side they cannot, decreasing the $30 \mathrm{p}$ production rate. As $v$ increases beyond $v_{c}$, the modes normal to the plate surface are switched on. The Einstein relationships between stimulated and spontaneous transition rates imply that spontaneous decay was similarly affected in this experiment. The experiment of Vaidyanathan et al. is a beautiful example of how Rydberg atoms are sensitive to their immediate environment, making them the perfect testbed for what would otherwise be minute and unmeasurable effects. 